\title{
Evaluation of the severity of ulcerative colitis using endoscopic dual red imaging targeting deep vessels
}

\section{다 (ㄱ) $\ominus$}

\author{
Authors \\ Makoto Naganuma ${ }^{1,2}$, Naohisa Yahagi ${ }^{3}$, Rieko Bessho ${ }^{1}$, Keiko Ohno $^{1}$, \\ Mari Arai ${ }^{1}$, Makoto Mutaguchi', Shinta Mizuno ${ }^{1}$, Ai Fujimoto ${ }^{3}$, \\ Toshio Uraoka ${ }^{3}$, Masayuki Shimoda ${ }^{4}$, Naoki Hosoe ${ }^{2}$, Haruhiko \\ Ogata $^{3}$, Takanori Kanai ${ }^{1}$ \\ Institutions \\ 1 Department of Gastroenterology and Hepatology, Keio \\ University, Tokyo, Japan \\ 2 Center for Diagnostic and Therapeutic Endoscopy, Keio \\ University, Tokyo, Japan \\ 3 Cancer Center, Keio University, Tokyo, Japan \\ 4 Division of Diagnostic Pathology, Keio University, Tokyo, Japan
}

submitted 9.5.2016

accepted after revision 4.10.2016

Bibliography

DOI http://dx.doi.org/10.1055/s-0042-119393 |

Endoscopy International Open 2017; 05: E76-E82

(c) Georg Thieme Verlag KG Stuttgart · New York

ISSN 2364-3722

Corresponding author

Makoto Naganuma, MD, PhD, Division of Gastroenterology and

Hepatology, School of Medicine, Keio University, 35 Shinanomachi,

Shinjuku-ku, Tokyo, 160-8582, Japan

Fax: +81-3-3341-3631

maknaganuma@gmail.com

takagast@z7.keio.jp

\section{ABSTRACT}

Background and study aims Colonoscopies can predict long-term prognoses in patients with ulcerative colitis (UC). Recently, a new imaging technology has been developed that uses 3 types of illumination with center wavelengths of $540 \mathrm{~nm}, 600 \mathrm{~nm}$, and $630 \mathrm{~nm}$. The use of both the $600-\mathrm{nm}$ and 630 -nm lights (Dual red imaging; DRI) is critical for identifying blood vessels in deeper tissue. The aim of this study was to evaluate the usefulness of DRI for assessing the severity of inflammation in patients with UC.

Patients and methods A total of 43 UC patients were retrospectively enrolled to evaluate the endoscopic severity of 112 colon segments, and Mayo endoscopic scores, DRI scores and the severity of inflammation on a visual analogue scale (VAS) were compared. The Mayo endoscopic scores, DRI scores, and histologic scores were evaluated, and the interobserver agreement on DRI scores among 5 investigators was also assessed. The usefulness of DRI scores for predicting prognoses was also assessed in patients with clinical remission.

Results The DRI scores were closely correlated with the VAS for the severity of colonic inflammation $(r=0.96)$ and the histologic scores $(r=0.72-0.8)$. The DRI scores had a higher rate of interobserver agreement $(\mathrm{K}$ values $=0.63-0.88)$ than the Mayo endoscopic scores $(\kappa$ values $=0.44-0.59)$. Inter-observer agreement between 4 non-experts was also excellent (mean $\mathrm{k}$ value $=0.76$, range 0.63 0.82 ). The expected time until recurrence was significantly longer in patients with lower DRI scores $(P<0.01)$.

Conclusion DRI can be used in patients with mild to moderate endoscopic severity because it targets the deep vascular pattern. The prognosis of UC can be predicted by assessing deep vessels using DRI.

\section{Introduction}

Narrow-band imaging (NBI) was developed to enhance the contrast of the capillary pattern in the superficial layer of the gastrointestinal tract [1-5]. NBI at $415 \mathrm{~nm}$ is a useful method to support endoscopic observations of early stage cancers, such as esophageal cancer [3]. The combination of the NBI technique with magnification endoscopy is useful for detecting early gastrointestinal cancers and for estimating the depth of cancer invasion by assessing vascular and surface patterns [4, 5]. In active ulcerative colitis (UC), however, the vascular pattern seen using NBI is not as clear (\Supplemental Fig. 1).

Recently, a new imaging technology has been introduced that uses 3 wavelengths for illumination: $540 \mathrm{~nm}, 600 \mathrm{~nm}$ and $630 \mathrm{~nm}$. The $600-\mathrm{nm}$ and $630-\mathrm{nm}$ lights are critical for identifying blood vessels in the submucosal tissue and bleeding points.
Thus, dual red imaging (DRI) could be a promising way to avoid severe bleeding during endoscopic submucosal resection for the treatment of early stage gastrointestinal tumors $[6,7]$. With respect to the identification of blood vessels in the submucosal tissue, DRI may detect deeper vessels in UC patients ( Supplemental Fig.1c). Because DRI can primarily enhance vessel patterns, we hypothesized that, by using DRI, differences among investigators' assessments of the severity of inflammation would be minimal. However, no study has evaluated the usefulness of DRI in UC patients.

The aim of this study was to evaluate the usefulness of DRI for assessing the severity of inflammation in patients with active UC. The assessment of UC lesions in patients with mild or moderate disease is important for assessing the severity of inflammation, and discrepancies among investigators have been 
observed in UC. Therefore, we evaluated the usefulness of DRI in UC patients with mild to moderate endoscopic severity.

\section{Patients and methods}

\section{Patients}

From March 2012 to April 2013, a total of 43 patients previously diagnosed with UC at the outpatient department of Keio University Hospital were enrolled in this study. All UC patients were diagnosed according to the criteria of the Research Committee on Inflammatory Bowel Disease in Japan [8]. Patients with fulminant disease were excluded because they required immediate intensive medical therapeutics, hospitalization, or surgery. This study was retrospectively conducted, and the clinical indices were prospectively collected during regular visits.

\section{Dual-red imaging}

This new imaging technology uses illumination at 3 wavelengths: $540 \mathrm{~nm}, 600 \mathrm{~nm}$ and $630 \mathrm{~nm}$. With respect to the identification of blood vessels in the submucosal tissue, the $600-\mathrm{nm}$ and 630-nm lights can penetrate the deeper tissue because the scattering properties of these lights are minimal. Furthermore, light absorption by blood vessels is much stronger at $600 \mathrm{~nm}$ than at $630 \mathrm{~nm}$. Therefore, the intensity of light reflected by blood vessels at $630 \mathrm{~nm}$ is greater than that at $600 \mathrm{~nm}$. This difference allows detection of blood vessels in the deeper tissue using the intensity balance of each wavelength of reflected light rather than white-light imaging (WLI) and DRI. The mechanism underlying how blood flow and bleeding can be more easily identified using DRI compared with conventional imaging methods is being actively studied.

\section{Colonoscopy}

Total ileocolonoscopies (ICS) were performed on all 43 patients. All patients were required to drink $2000 \mathrm{~mL}$ to $3000 \mathrm{~mL}$ of polyethylene glycol (Ajinomoto Farma, Co, Ltd, Tokyo, Japan) before the procedure. Pethidine hydrochloride (Tanabe-Mitsubishi, Tokyo, Japan) was administered immediately prior to ICS to reduce patient burden. All colonoscopy images were recorded on a digital versatile disc. Physicians could instantaneously switch from WLI to DRI by pressing a button on the control handle of the colonoscope. WLI and DRI recordings were taken in the ascending colons, sigmoid colons, and rectums of all 43 patients. To reduce the risk of bleeding caused by biopsies, we evaluated the severity of inflammation for only 3 colonic segments. In all cases, at each segment, the WLI was recorded first, and then the DRI was recorded at the same lesions. A total of 129 videos were collected, and 17 videos were excluded due to insufficient bowel cleaning. Finally, 112 videos (37 from the ascending colon, 35 from the sigmoid colon, 40 from the rectum) were collected to evaluate the endoscopic severity of inflammation using WLI and DRI.

\section{Scoring the severity of inflammation}

Five endoscopists independently evaluated the severity of mucosal inflammation using the Mayo endoscopic score [9] for the WLI videos of the 112 segments of the colon. One was an ex- pert in diagnosis of UC (approximately 400 colonoscopies for UC annually), and the other 4 investigators were non-experts (50 cases annually). The endoscopic severity of inflammation seen using DRI was also evaluated using a novel DRI score. The Mayo score was classified as follows: below 0 , normal; 1 , erythema, decreased vascular pattern, mild friability; 2, marked erythema, absent vascular pattern, friability, erosion; 3, spontaneous bleeding, ulcerations. The DRI scores were classified as DRI 1, 2, 3, and 4. DRI1 was defined as a normal vascular pattern in both the superficial (brown) and deep (green) vessels. DRI2 was defined as the patchy or complete obliteration of the brown vessels with clear reorganization of the green vessels. DRI3 was defined as patchy obliteration of the green vessels, and DRI4 was defined as the complete obliteration of the green vessels. The overall severity was also assessed on a visual analogue scale (VAS). VAS scores ranged from 0 (completely normal) to 100 (most severe).

Histologic severity of inflammation was scored by a pathologist using the Geboes score [10] as follows: grade 0, structural and architectural changes; grade 1, chronic inflammatory infiltrate; grade 2, neutrophils and eosinophils in the lamina propria; grade 3, neutrophils in the epithelium; grade 4, crypt destruction; grade 5, erosions or ulceration. The pathologist was blinded to the clinical information.

\section{Assessment of clinical prognosis in patients with various DRI scores}

The usefulness of DRI scores for predicting clinical outcomes was assessed. Partial Mayo scores were recorded on the patients' medical charts at each visit to the clinic. Clinical remission was defined as a partial Mayo score $\leq 1$. The rate of clinical recurrence was analyzed for patients with each DRI score (from DRI1 to DRI4). Clinical recurrence was defined as a partial Mayo score $\geq 3$.

\section{Data collection and statistical assessment}

Our main endpoints in this study were as follows: 1) assessment of the correlation between the DRI score and the Mayo endoscopic score and between the DRI score and the histological grade, 2) inter-observer agreement on the DRI score and the Mayo endoscopic score, and 3) assessment of the correlation between the DRI score and the rate of recurrence in patients with clinical remission.

We evaluated the correlation between the DRI score, the Mayo endoscopic score and the histopathological grade using Spearman's rank correlation coefficient. To validate the DRI score, interobserver agreement between 2 endoscopists was examined, and the kappa value was calculated. Clinical information, such as age, gender, duration of disease, disease type (first attack, relapsing/remission or chronic continuous) and the use of medical treatments (at the time of the colonoscopy and at each visit to the clinic), was collected. Although we did not schedule visits to the clinic, most patients visited our hospital every 8 to 12 weeks.

In patients with clinical remission, the association between the DRI score and the time until clinical recurrence was assessed using the Kaplan-Meier method, and the difference be- 


\begin{tabular}{|c|c|c|c|}
\hline \multicolumn{2}{|c|}{ Gender (male:female) } & 26:17 & \\
\hline \multicolumn{2}{|l|}{ Median age (years) } & 38.4 & $(24-72)$ \\
\hline \multicolumn{2}{|c|}{ Median duration of disease (years) } & 8.6 & $(0-23)$ \\
\hline \multirow[t]{4}{*}{ Extent of disease } & Total colitis & 18 & \\
\hline & Left-sided colitis & 8 & \\
\hline & Distal colitis & 9 & \\
\hline & Proctitis & 8 & \\
\hline \multirow[t]{3}{*}{ Type of disease } & First attack & 3 & \\
\hline & Relapse-remission & 39 & \\
\hline & Chronic disease & 1 & \\
\hline \multicolumn{2}{|c|}{ Mean partial Mayo score at entry } & 1.2 & $(0-6)$ \\
\hline \multirow[t]{5}{*}{ Current treatment } & No medication & 7 & \\
\hline & Oral 5-ASA & 36 & \\
\hline & Topical 5-ASA & 7 & \\
\hline & Topical steroid & 2 & \\
\hline & Thiopurine & 5 & \\
\hline \multicolumn{4}{|c|}{ 5-ASA, 5-aminosalicylate } \\
\hline
\end{tabular}

tween the groups was analyzed using the log-rank test and the Breslow test. All $P$ values are two-tailed, and $P<0.05$ was considered statistically significant. All statistical analyses were per- formed using Statistical Package for the Social Sciences software version 22.0 (SPSS Inc., Chicago, IL).

\section{Results \\ Patient characteristics at baseline}

The clinical features of the patients and their medications for UC are listed in $>$ Table 1 . Forty-three patients with UC were included; of them, 36 (84\%) and 5 (12\%) were taking oral 5-aminosalicylates and thiopurine, respectively, whereas 7 patients did not take any medication for UC. The mean partial Mayo score of this cohort was 1.2. Serum CRP levels ranged from 0.01 to $1.35 \mathrm{mg} / \mathrm{dL}$, and CRP levels were within the normal range (less than $0.35 \mathrm{mg} / \mathrm{dL}$ in our institution) in $84 \%$ of patients.

\section{Correlation between DRI score and VAS score for the severity of inflammation}

- Fig. 1 indicates the typical findings for each DRI score in patients with UC. A total of 112 segments were assessed for the severity of inflammation using the Mayo endoscopic score, the DRI score and the VAS score. The mean DRI and VAS scores were $2.9 \pm 1.2$ (range: $1-4$ ) and 25.0 \pm 18.1 (range 4.5-65.5), respectively. The distribution of severity scores using the VAS $(0-100$ points $)$ with DRI is presented in - Fig. 2 . The VAS score was significantly correlated with the DRI score $(r=0.957, P<$ $0.001)$. The median VAS score in patients scored as DRI1, 2, 3, and 4 was 6.5 (interquartile range, IQR=2.6), $18.0(I Q R=5.0)$, $35.0(\mathrm{IQR}=7.0)$, and $52.5(\mathrm{IQR}=8.3)$, respectively.

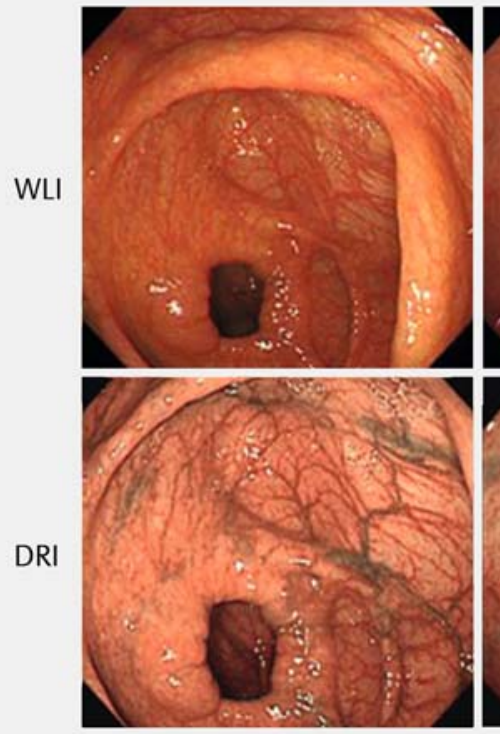

DRI 1

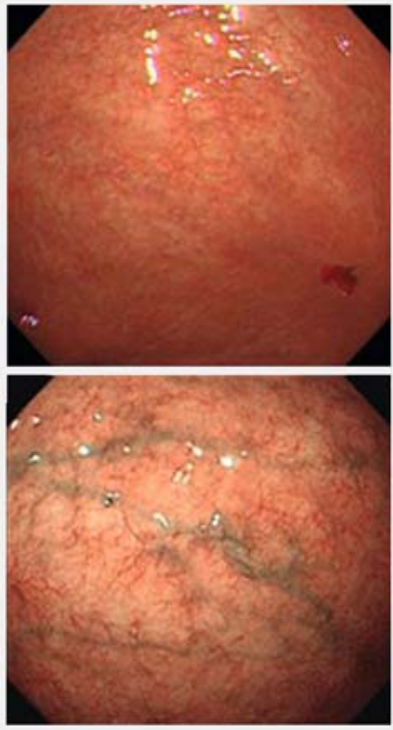

DRI 2

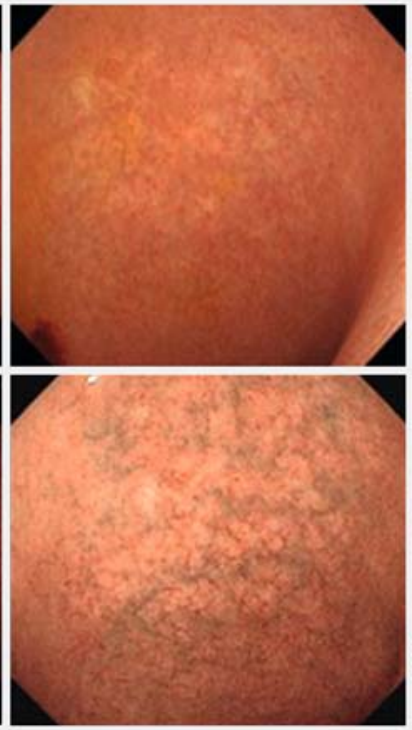

DRI 3

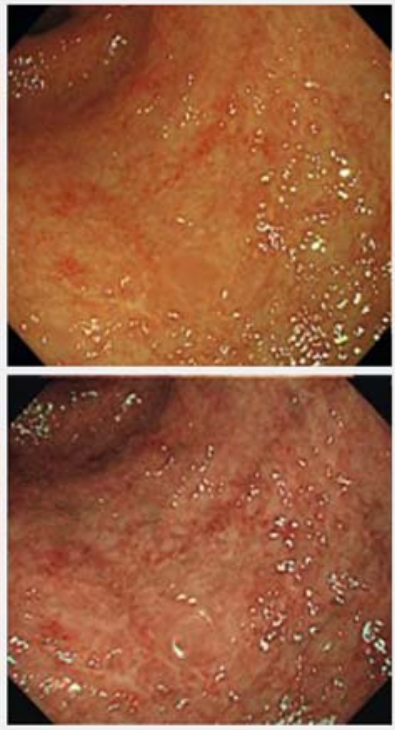

DRI 4

- Fig. 1 Typical findings for each DRI score. DRI1 was defined as a normal vascular pattern in both the superficial (brown) and deep (green) vessels. DRI2 was defined as patchy or complete obliteration of the brown vessels with clear reorganization of the green vessels. DRI3 was defined as the patchy obliteration of the green vessels, and DRI4 was defined as the complete obliteration of the green vessels. 


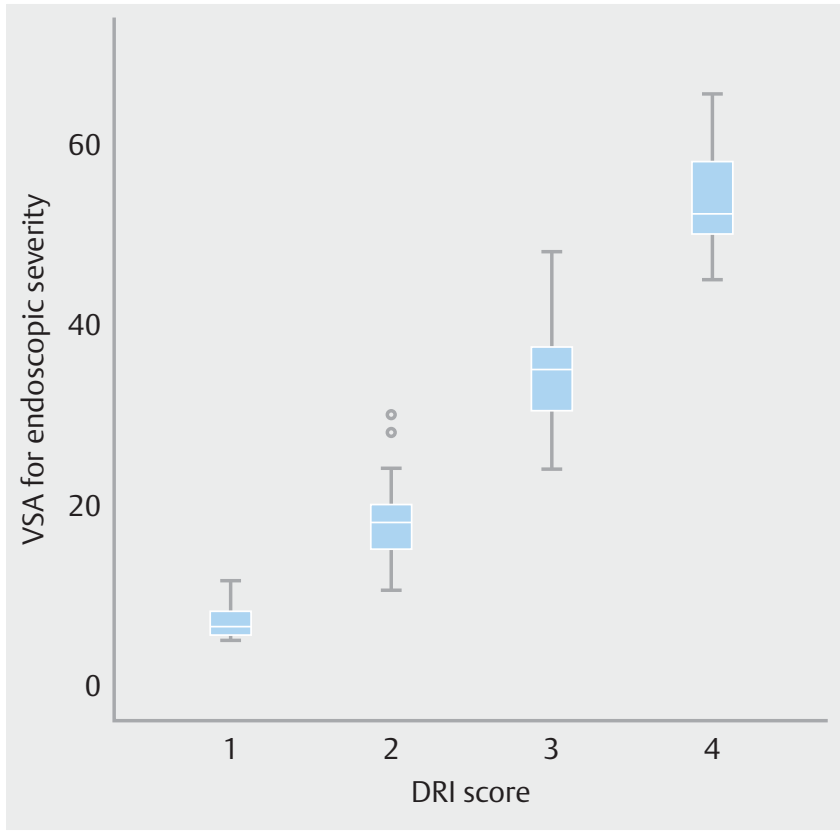

- Fig. 2 Relationship between DRI score and VAS score with respect to the severity of endoscopic inflammation. A) The DRI score and the VAS score were closely correlated $(r=0.957)$. b) Box plots of the VAS score for each DRI score. Only 2 scores were observed within $1.5-3 \mathrm{IQRs}$ of the third quartile (Q3) in patients with DRI = 2 (circle plot). Dotted line: median, Black line: range of VAS for each DRI score, Brown box: interquartile range (IQR).

\section{Correlation between DRI score, endoscopic score and histopathology}

Next, we assessed the relationship between the DRI score and the Mayo endoscopic score. As $>$ Table 2 indicates, the DRI score was significantly correlated with the Mayo endoscopic score in analyses by both experts and non-experts $(r=0.695-$ $0.778 ; P<0.001$ in each investigator). The relationship between the DRI score and the histological grade was significantly similar for each investigator ( $\triangleright$ Table 3 ).

\section{Interobserver agreement was better for the DRI score than for the Mayo endoscopic score}

The $\mathrm{k}$ value validating the interobserver agreement on the DRI score was moderate to excellent (mean $\mathrm{K}$ value $=0.76$, range $0.63-0.88)$, as $>$ Table 4 a indicates. Interestingly, inter-observer agreement was even excellent among the analyses from 4 non-experts (mean $\mathrm{k}$ value $=0.76$, range $0.63-0.82$ ). However, relatively lower inter-observer agreement among the 5 investigators was observed for the Mayo endoscopic score assessments, with a mean $\mathrm{K}$ value of 0.53 ( $\sim$ Table $4 \mathbf{b}$ ), and this agreement was significantly lower than the agreement on DRI scores $(P<0.001)$. Intra-observer agreement values for the Mayo endoscopic score and the DRI score were comparable (the $\mathrm{k}$ values were 0.89 and 0.91 , respectively).
- Table 2 Correlation between DRI scores and Mayo endoscopic scores.

\begin{tabular}{|l|l|r|}
\hline & DRI score vs. Mayo endoscopic score & P value \\
\hline Expert & $r=0.778$ & $<0.001$ \\
\hline Non-expert 1 & $r=0.695$ & $<0.001$ \\
\hline Non-expert 2 & $r=0.762$ & $<0.001$ \\
\hline Non-expert 3 & $r=0.720$ & $<0.001$ \\
\hline Non-expert 4 & $r=0.760$ & $<0.001$ \\
\hline
\end{tabular}

- Table 3 Correlation between endoscopic score and Geboes' histologic score.

\begin{tabular}{|l|l|r|}
\hline & DRI score vs. Geboes score & P value \\
\hline Expert & $r=0.821$ & $<0.001$ \\
\hline Non-expert 1 & $r=0.792$ & $<0.001$ \\
\hline Non-expert 2 & $r=0.763$ & $<0.001$ \\
\hline Non-expert 3 & $r=0.740$ & $<0.001$ \\
\hline Non-expert 4 & $r=0.840$ & $<0.001$ \\
\hline
\end{tabular}

- Table4 Interobserver agreement ( $k$ values) for DRI scores (a) and Mayo endoscopic scores (b).

\begin{tabular}{|l|l|l|l|l|}
\hline & Expert & $\begin{array}{l}\text { Non- } \\
\text { expert 1 }\end{array}$ & $\begin{array}{l}\text { Non- } \\
\text { expert 2 }\end{array}$ & $\begin{array}{l}\text { Non- } \\
\text { expert 3 }\end{array}$ \\
\hline a) & & & \\
\hline Non-expert 1 & 0.875 & & & \\
\hline Non-expert 2 & 0.874 & 0.824 & & \\
\hline Non-expert 3 & 0.763 & 0.712 & 0.702 & \\
\hline Non-expert 4 & 0.815 & 0.717 & 0.692 & 0.630 \\
\hline b) & & & & \\
\hline Non-expert 1 & 0.590 & & & \\
\hline Non-expert 2 & 0.542 & 0.566 & & \\
\hline Non-expert 3 & 0.535 & 0.505 & 0.519 & \\
\hline Non-expert 4 & 0.575 & 0.54 & 0.505 & 0.440 \\
\hline
\end{tabular}

\section{DRI score and the prediction of prognosis in patients with clinical remission}

We next assessed whether endoscopic severity assessed using DRI at baseline was associated with an increased risk of clinical recurrence among 35 patients in clinical remission. In this analysis, the highest DRI score (the most severe score) among the segments was used. Over a mean follow-up of $25.3 \pm 9.3$ months, 9 patients $(43 \%)$ experienced clinical recurrence, whereas the other 26 patients remained in clinical remission. No recurrence was observed in patients scored as DRI1. The recurrence rates in patients scored as DRI2, 3, or 4 were $15 \%$ 


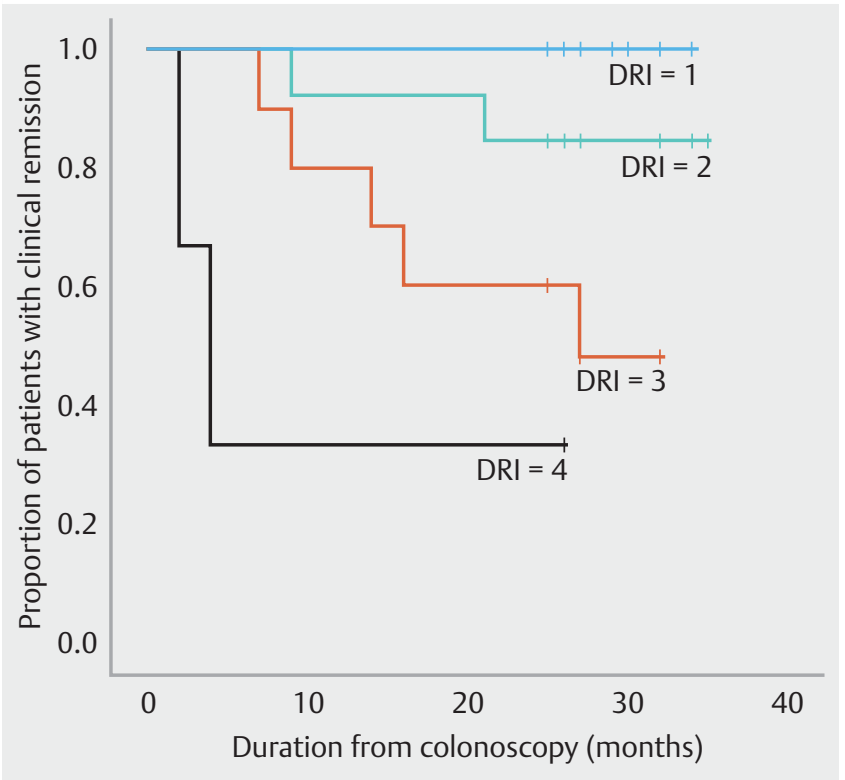

- Fig. 3 Non-recurrence rates in patents with various DRI scores that were in clinical remission at baseline. The expected time until recurrence was significantly longer in patients with lower DRI scores (log-rank test, Breslow test $P=0.002$ ).

(2/13), $50 \%(5 / 10)$, and $67 \%(2 / 3)$, respectively. The expected time until recurrence was significantly longer in patients with lower DRI scores ( $\triangleright$ Fig. 3, log-rank test, Breslow test $P=$ $0.002)$. When patients were divided into a lower DRI group (DRI 1 -2) and a higher DRI group (DRI 3-4), the mean expected time until recurrence was 33.2 months $(95 \% \mathrm{Cl} 30.7-35.7$ ) in the lower DRI group and 20.7 months ( $95 \% \mathrm{Cl} 14.3-27.1)$ in the higher DRI group.

\section{Discussion}

UC is a chronic inflammatory bowel disease characterized by blood in the stool, diarrhea, abdominal pain, and extra-intestinal symptoms. Endoscopy plays a pivotal role in the assessment of the severity of UC, and endoscopic assessment is a critical endpoint for assessing the efficacy of medications. Endoscopic remission gives an improved prognosis in patients with UC and is considered a treatment goal [11]. In patients with newly diagnosed UC who were treated with steroids, the rates of hospitalization, the need for immunosuppression therapy, and the need for colectomy were significantly higher in patients with clinical remission without mucosal healing than in those with both clinical and endoscopic remission [12]. Mucosal healing is an independent predictor of long-term outcomes from infliximab (IFX) treatment in patients with UC $[13,14]$. A recent study indicated that the degree of mucosal healing after 8 weeks of IFX use was correlated with improved clinical outcomes, including a reduced incidence of colectomy [14]. In this study, the medium-term prognosis (30 weeks after the starting on IFX) was better in patients with Mayo endoscopic scores of 0-1 than in those with Mayo endoscopic scores of 2 - 3. Furthermore, significant differences between Mayo endoscopic scores of 0 and 1 that were detected 8 weeks after start- ing on IFX could predict clinical remission at week 30 . These results suggest that endoscopy is critical for assessing the severity of inflammation and can predict medium- to long-term prognoses in UC patients after the onset of remission.

Although original and modified Baron scores $[15,16]$ and the Mayo score for proctosigmoidoscopy [17] have been widely used and are easy for investigators to use, recent studies have demonstrated that the rate of agreement is only $27 \%$ for endoscopic remission (Baron score 0 ) and $37 \%$ for moderate activity (Baron 2). Inter-observer disagreement on the severity of inflammation has been reported for UC [18]. Thus, the development of new methods/techniques to assess severity is warranted. More recently, the Ulcerative Colitis Endoscopic Index of Severity (UCEIS) and the Ulcerative Colitis Colonoscopic Index of Severity (UCCIS) were developed to solve the problem of low interobserver agreement on endoscopic scores [18-20]. An assessment of vascular patterns including both endoscopic indices revealed excellent interobserver agreement for the vascular pattern ( $\mathrm{k}$ value $=0.74-0.86$ ), and the intraobserver agreement for the vascular pattern $(\kappa$ value $=0.87$ ) was better than that for bleeding ( $\mathrm{k}$ value $=0.47$ ).

DRI was developed to easily identify blood vessels in deeper tissue. In this study, the usefulness of DRI for assessing the severity of UC was investigated by focusing on only the mucosal and submucosal vessels. Usually, the vascular pattern in a conventional colonoscopy is partially identifiable or is absent in cases of mild to moderate UC inflammation. DRI can primarily enhance the vessel pattern and the identification of deep vessels. Importantly, deep vessels can be detected even when the vascular pattern is partially visible or has disappeared using WLI. Therefore, by targeting the deep vascular pattern, DRI can be used in patients with mild to moderate endoscopic severity. Because DRI enhances the vascular pattern, inter-observer disagreement might be minimal. In fact, our study indicated that the $\mathrm{k}$ values for the DRI scores between 2 investigators were better than those for the previously used endoscopic score. Furthermore, our study also indicated that the clinical prognosis is better in patients with deep vessels observed using DRI $(D R I=2)$ than in patients with partially visible vessels (DRI = 3). Thus, DRI is useful for predicting the middle- to long-term prognosis of UC patients. Furthermore, DRI could be used to evaluate colonic inflammation after beginning intensive medical treatment (e.g., anti-TNF agents, steroids) when the vessel pattern is absent using conventional WLI.

There are some limitations to this study. The investigators observed videos of WLI before DRI in all cases. Thus, assessment of severity based on DRI was affected by the WLI. In this study, we did not use high-definition endoscopy. However, our recent preliminary study using high-definition endoscopy indicated that the interobserver agreement on Mayo endoscopic scores was not as high (data not shown, $\mathrm{k}$ value $=0.66$ ) compared with that using DRI. Furthermore, a pathologist assessed the histopathological severity in this study, therefore, inter-observer validation of the histopathological assessment was not considered. The usefulness of DRI for UC should be assessed in a larger study. Finally, in this study, we did not assess the endoscopic usefulness of white light imaging in predicting clinical 
outcomes using endoscopic indices such as the Mayo score. A previous study indicated that a Mayo endoscopic score of 0 (complete endoscopic remission) predicted a better clinical outcome, and significant differences have been identified between a Mayo endoscopic score of 0 and 1 (mild endoscopic severity). However, a Mayo endoscopic score of 1 was observed in almost $70 \%$ of the patients in our cohort. Thus, we did not confirm the significant differences that have been identified between a MES of 0 and 1 in this study. Importantly, DRI could separate patients with the same Mayo endoscopic score into groups with DRI scores of 2 or DRI scores of 3-4.

\section{Conclusion}

In conclusion, DRI is a novel, simple tool for assessing the severity of inflammation in UC patients. The DRI score was correlated with the VAS score for the severity of colonic inflammation and exhibited a high rate of inter-observer agreement. The DRI score was closely correlated with both the endoscopic and the histological grades. The prognosis of UC can be predicted by assessing deep vessels using DRI.

\section{Competing Interests}

Colonoscopy with DRI capability was provided by the Olympus company. However, the company representative did not contribute either to the performance or the planning of this study, and did not write or help to write the manuscript.

\section{References}

[1] Yoshida T, Inoue H, Usui S et al. Narrow-band imaging system with magnifying endoscopy for superficial esophageal lesions. Gastrointest Endosc 2004; 59: 288 - 295

[2] Gono K, Obi T, Yamaguchi M et al. Appearance of enhanced tissue features in narrow-band endoscopic imaging. J Biomed Opt 2004; 9: $568-577$

[3] Muto M, Minashi K, Yano T et al. Early detection of superficial squamous cell carcinoma in the head and neck region and esophagus by narrow band imaging: a multicenter randomized controlled trial. Clin Oncol 2010; 28: 1566-1572

[4] Sano Y, Horimatsu T, Fu KI et al. Magnifying observation of microvascular architecture of colorectal lesions using a narrow band imaging system. Dig Endosc 2006; 18: 44-S51
[5] Katagiri A, Fu KI, Sano Y et al. Narrow band imaging with magnifying colonoscopy as diagnostic tool for predicting histology of early colorectal neoplasia. Aliment Pharmacol Ther 2008; 27: 1269-1274

[6] Yahagi N. Dual Red Imaging; A new endoscopic imaging technology for clear visualization of bleeding points in endoscopic submucosal dissection. Gastrointestinal Endosc 2014: A1192

[7] Horii J, Uraoka T, Goto O et al. Dual Red Imaging; A new endoscopic imaging technology for clear visualization of thick blood vessels in deeper tissue and bleeding points. Gastrointestinal Endosc 2014: A3116

[8] Yao T, Matsui T, Hiwatashi N. Crohn's disease in Japan: diagnostic criteria and epidemiology. Dis Colon Rectum 2000; 43: 85 - S93

[9] Schroeder KW, Tremaine W], Ilstrup DM. Coated oral 5-aminosalicylic acid therapy for mildly to moderately active ulcerative colitis. A randomized study. N Engl J Med 1987; 317: 1625 - 1629

[10] Geboes K, Riddell R, Ost A et al. A reproducible grading scale for histological assessment of inflammation in ulcerative colitis. Gut 2000; 47: $404-409$

[11] Neurath MF, Travis SP. Mucosal healing in inflammatory bowel diseases: a systematic review. Gut 2012; 61: 1619-1635

[12] Ardizzone S, Cassinotti A, Duca P et al. Mucosal healing predicts late outcomes after the first course of corticosteroids for newly diagnosed ulcerative colitis. Clin Gastroenterol Hepatol 2011; 9: 483-489

[13] Arias MT, Vande Casteele N, Vermeire S et al. A panel to predict longterm outcome of infliximab therapy for patients with ulcerative colitis. Clin Gastroenterol Hepatol 2015; 13: 531-538

[14] Colombel JF, Rutgeerts P, Reinisch W et al. Early mucosal healing with infliximab is associated with improved long-term clinical outcomes in ulcerative colitis. Gastroenterology 2011; 141: 1194 - 1201

[15] Baron JH, Connell AM, Lennard-Jones JE. Variation between observers in describing mucosal appearances in proctocolitis. Br Med J 1964; 1 : $89-99$

[16] Hawthorne AB, Logan RF, Hawkey C] et al. Randomised controlled trial of azathioprine withdrawal in ulcerative colitis. BMJ 1992; 305: 20-22

[17] Schroeder KW, Tremaine W], Ilstrup DM. Coated oral 5-aminosalicylic acid therapy for mildly to moderately active ulcerative colitis. A randomized study. N Engl J Med 1987; 317: 1625-1629

[18] Travis SP, Schnell D, Krzeski P et al. Developing an instrument to assess the endoscopic severity of ulcerative colitis: the Ulcerative Colitis Endoscopic Index of Severity (UCEIS). Gut 2012; 61: 535-544

[19] Travis SP, Schnell D, Krzeski P et al. Reliability and initial validation of the ulcerative colitis endoscopic index of severity. Gastroenterology 2013; 145: 987 - 995

[20] Samuel S, Bruining DH, Loftus EVJr et al. Validation of the ulcerative colitis colonoscopic index of severity and its correlation with disease activity measures. Clin Gastroenterol Hepatol 2013; 11: 49-54 

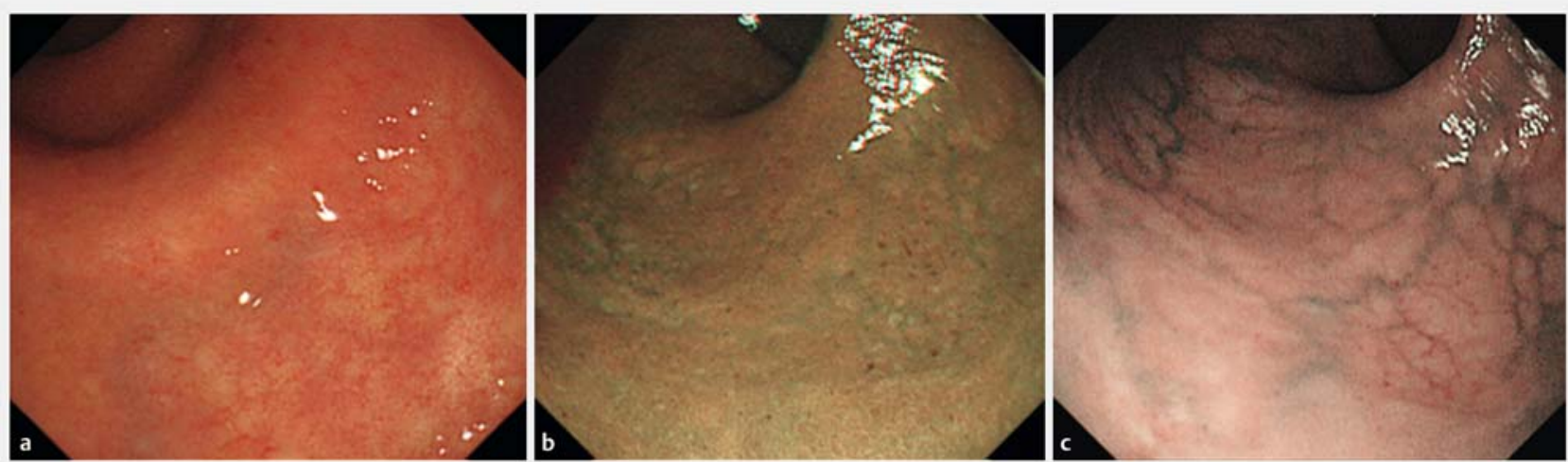

- Supplemental Fig. 1 Rectal mucosa in patients with mild inflammation. a The vessel pattern was obliterated using white light imaging, and b vessel information was not observed using narrow-band imaging). c Dual-red imaging indicated that the superficial vasculature was patchy (brown), and deep vessels (green) were also observed. 